University of Nebraska - Lincoln

DigitalCommons@University of Nebraska - Lincoln

Special Education and Communication

Disorders Faculty Publications
Department of Special Education and Communication Disorders

2010

\title{
Kinematics of disease progression in bulbar ALS
}

\author{
Yana Yunusova \\ University of Toronto, Faculty of Law, yana.yunusova@utoronto.ca \\ Jordan R. Green \\ University of Nebraska-Lincoln, jgreen4@unl.edu \\ Mary J. Lindstrom \\ University of Wisconsin-Madison \\ Laura J. Ball \\ University of Nebraska Medical Center-Omaha \\ Gary L. Pattee \\ University of Nebraska Medical Center-Omaha \\ See next page for additional authors
}

Follow this and additional works at: https://digitalcommons.unl.edu/specedfacpub

Part of the Special Education and Teaching Commons

Yunusova, Yana; Green, Jordan R.; Lindstrom, Mary J.; Ball, Laura J.; Pattee, Gary L.; and Zinman, Lorne, "Kinematics of disease progression in bulbar ALS" (2010). Special Education and Communication Disorders Faculty Publications. 5.

https://digitalcommons.unl.edu/specedfacpub/5

This Article is brought to you for free and open access by the Department of Special Education and Communication Disorders at DigitalCommons@University of Nebraska - Lincoln. It has been accepted for inclusion in Special Education and Communication Disorders Faculty Publications by an authorized administrator of DigitalCommons@University of Nebraska - Lincoln. 


\section{Authors}

Yana Yunusova, Jordan R. Green, Mary J. Lindstrom, Laura J. Ball, Gary L. Pattee, and Lorne Zinman 
Published in Journal of Communication Disorders 43:1 (2010), pp. 6-20; doi: 10.1016/j.jcomdis.2009.07.003

Copyright $@ 2009$ Elsevier Inc. Used by permission. http://www.elsevier.com/locate/jcomdis

Submitted September 11, 2008; revised May 21, 2009; accepted July 15, 2009; published online July 24, 2009.

\title{
Kinematics of disease progression in bulbar ALS
}

\author{
Yana Yunusova, ${ }^{1}$ Jordan R. Green, ${ }^{2}$ Mary J. Lindstrom, ${ }^{3}$ \\ Laura J. Ball, ${ }^{4}$ Gary L. Pattee, ${ }^{4}$ and Lorne Zinman ${ }^{1,5}$ \\ 1 University of Toronto \\ 2 University of Nebraska-Lincoln \\ 3 University of Wisconsin-Madison \\ 4 University of Nebraska Medical Center-Omaha \\ 5 Sunnybrook Health Science Centre, Toronto \\ Corresponding author - Y. Yunusova, Department of Speech and Language Pathology, \\ Rehabilitation Sciences Building, University of Toronto, 160-500 University Avenue, \\ Toronto, ON M5G 1V7, Canada; tel 416 978-6890, fax 416 978-1596, \\ email yana.yunusova@utoronto.ca
}

\begin{abstract}
The goal of this study was to investigate the deterioration of lip and jaw movements during speech longitudinally in three individuals diagnosed with bulbar amyotrophic lateral sclerosis (ALS). The study was motivated by the need to understand the relationship between physiologic changes in speech movements and clinical measures of speech performance such as speaking rate and speech intelligibility. Movements of the lip and jaw were quantified with respect to their size speed, and duration. The data revealed several changes in lip and jaw movement that coincided with ALS progression. In two out of three speakers, the changes in measures of path distance and speed anticipated the drop in speech intelligibility by approximately 3 months. With disease progression, increases in movement duration coincided with declines in speech intelligibility. Overall, the movement measures appeared to be sensitive to disease progression in ALS.

Learning outcomes: By the end of the article, the reader should be able to: (1) describe the changes that occur in articulatory movements of the jaw and lower lip in ALS; (2) understand the relationship between physiologic measures of movement and speech intelligibility and speaking rate; (3) identify critical points in the disease progression and understand which quantitative measures reveal the state of the bulbar system at these time points.
\end{abstract}

\section{Introduction}

Amyotrophic lateral sclerosis (ALS) is a motor neuron disease characterized by progressive degeneration of motor neurons in the brain, brainstem, and spinal cord. Degeneration of the upper and lower motor neurons leads to spasticity, impaired reflexes, muscle fatigue, muscle weakness, and eventually atrophy. Affected individuals vary significantly in the locus of disease onset, presentation at diagnosis, and rate of progression (Brooks et al., 1991). ALS is a terminal disease associated with short survival; the median survival post-diagnosis is approximately 30 months (Millul et al., 2005). Shorter survival times (under 20 months) are associated with the presence of bulbar (speech and swallowing), as opposed to purely spinal 
(limb), symptoms at disease onset and at diagnosis (Czaplinski, Yen, \& Apperl, 2006). Because ALS progresses so rapidly in many individuals, an important goal of clinical management is to anticipate functional changes in patients' performance in order to teach new communication strategies and compensatory skills (e.g., assistive technology) before the patient's ability to learn these skills is impacted by the severity of their condition.

The motor denervation of bulbar musculature in ALS results in deterioration and eventual loss of the ability to swallow and communicate orally. Perceptual and acoustic features of dysarthria in ALS have been well studied (Darley, Aronson, \& Brown, 1975; see Duffy, 2005 for a comprehensive summary). Dysarthria in ALS is commonly characterized by reduced speaking and articulatory rates. The decrease in rate is often associated with increased pause time and increased segment durations, particularly for vowel sounds (Green et al., 2004; Tjaden and Turner, 2000; Turner and Weismer, 1993). Spectral properties of vowels and consonants (e.g., formant frequencies, transition extents and slopes, fricative spectral moments) are also affected by the disease, with vowels becoming more centralized and the frequency spectrum of consonants becoming less distinct (Kent et al., 1989; Kent et al., 1990; Kent et al., 1992; Tjaden and Turner, 1997; Turner et al., 1995; Weismer et al., 1988; Weismer et al., 1992; Weismer et al., 2001). These acoustic findings have been presumed to be due to the disease-related reduction and slowing of articulatory movements (see Caruso and Burton, 1987; Weismer et al., 1992).

Articulatory findings, although very limited, support this interpretation. One of the early studies of articulatory kinematics in two speakers with ALS demonstrated slowed articulatory movements, decreased displacement of the tongue and lip, but exaggerated displacements of the jaw during diadokokinetic tasks (Hirose, Kiritani, \& Sawasima, 1982). A recent study of articulatory movements in a group of nine speakers with ALS revealed that articulatory speed during vowels was consistently impaired across all oral articulators (Yunusova, Weismer, Westbury, \& Lindstrom, 2008). Aberrant displacements were found to be word and vowel-dependent and were more consistently present in movements of the tongue than other articulators and occasionally in the jaw. The jaw displacements were smaller than normal in words requiring larger articulator movements (e.g., consonant plus low vowel) but were larger than normal in words that only required relatively small jaw movements (e.g., consonant plus high vowel), suggesting difficulty in scaling of the vowel-related movements. These contradictory findings between studies underscore the need for additional research on the effects of ALS on jaw movements during speech.

The current study reports jaw and lower lip (+jaw) movement and intelligibility data collected longitudinally for approximately a year from three speakers with ALS. In the past, longitudinal studies documented the decline in speech intelligibility and speaking rate (DePaul and Kent, 2000; Kent et al., 1992; Mulligan et al., 1994; Nishio and Niimi, 2000; Yorkston et al., 1998). Some studies have also identified several acousticbased speech markers of disease progression (Kent et al., 1991; Mulligan et al., 1994; Ramig et al., 1990). To our knowledge, measures of articulatory kinematics have not yet been studied longitudinally.

Longitudinal studies are advantageous when dealing with heterogeneous populations, as in the case of ALS, since each participant can serve as their own control. Additionally, longitudinal investigations are necessary when the goal of research is to identify early predictors of future change. Because a person's ability to communicate orally is typically assessed based on speech intelligibility, anticipating the decline in intelligibility in a sensitive way is critical for timely clinical management of bulbar ALS. Yorkston and colleagues reported that speaking rate was a reliable predictor of speech intelligibility decline (Yorkston, Strand, Miller, Hillel, \& Smith, 1993). In their retrospective study of more than a hundred clinical cases, the authors discovered a rapid deterioration in speech intelligibility shortly after a decline in speaking rate to 100-120 words per minute (WPM). This finding was replicated by Ball and colleagues across a large group of patients with varying degrees of severity of bulbar symptoms (Ball, Beukelman, \& Pattee, 2002; see also Nishio \& Niimi, 2000). The authors suggested that the decline of speaking rate to 100-120 WPM should serve as a clinical indicator for initiation of an intervention focused on using assistive technology to support communication.

The main reason for the current study is to test the feasibility of using kinematic measures as early predictors of intelligibility decline. Although the sensitivity of measures of speech movements to disease onset and progression has not been determined, other physiologic measures of bulbar muscle performance, such as strength or rate of contractions, are known to be sensitive to motor neuron loss secondary to ALS. These measures, however, do not predict functional performance decline of the bulbar system as a whole, as indexed by speech intelligibility (DePaul and Brooks, 1993; Dworkin, 1980; Langmore and Lehman, 
1994). In contrast to oral strength measures, oral movement measures are obtained during speaking and, therefore, might show a direct relationship to speech intelligibility. This method also allows the tracking of individual articulators, which is needed to discern differential impairment among components of the bulbar system in ALS (DePaul et al., 1988; DePaul and Abbs, 1987; DePaul and Brooks, 1993; Hirose et al., 1982; Yunusova et al., 2008).

This pilot project on three individuals with bulbar ALS has two objectives: (1) to determine if speech movements might be affected earlier in the disease than measures of speaking rate and intelligibility, and (2) to determine the possibility of relations between longitudinal changes in articulation (jaw and lip), speech intelligibility, and speaking rate. Based on limited movement data reported in the ALS literature, we hypothesize that articulatory kinematics (e.g., movement amplitude and speed) will change over the course of disease progression. Furthermore, we hypothesize that the change in movement measures will anticipate the deterioration in speech intelligibility, thus serving as a potential early indicator of disease onset in the bulbar system.

\section{Methods}

\subsection{Participants}

Three male speakers from whom longitudinal data were available were studied in this pilot project. All participants were diagnosed with ALS by a clinical neurologist who specializes in neuromuscular disease (5th author), using the revised El Escorial criteria (Brooks, Miller, Swash, \& Munsat, 2000). All speakers had dysarthria at the time of study initiation; however, its severity varied between patients. None of the speakers had a significant history of speech, language, hearing, vision or neurological problems prior to the onset of ALS. All patients were taking low doses $(30-60 \mathrm{mg}$ ) of an experimental compound, $R(+)$ pramipexole, riluzole, vitamins, and various health supplements. Participant characteristics such as age, gender, time course of the disease, and ALSFRS-R scores (Cedarbaum et al., 1999) are summarized in Table 1.

\subsection{Speech sample}

The recorded task consisted of four sentences read at a comfortable reading rate and loudness and repeated five times in a fixed order. The sentences were Bobby is up to bat, Fast foxes have food feasts, Go give cake to Kate, and Wait for me a little while. Five repetitions of each highlighted word (i.e., bat, fast, feasts,

Table 1. Pertinent participant information. The clinical description of dysarthria was given by a single clinician $\left(3^{\text {rd }}\right.$ author).

\begin{tabular}{|c|c|c|c|c|c|}
\hline ID, sex, age & Characteristics & Session 1 & Session 2 & Session 3 & Session 4 \\
\hline $\mathrm{A} 1, \mathrm{M}, 44$ & $\begin{array}{l}\text { Ms post-onset } \\
\text { ALSFRS-R } \\
\text { Intelligibility } \\
\text { Speech Characteristics }\end{array}$ & \multicolumn{4}{|c|}{$\begin{array}{l}\text { Slightly strained voice (Sessions 1-2) progressed to moderately } \\
\text { severe dysarthria (Sessions 3-4) characterized by monopitch, } \\
\text { hypernasality, imprecise articulation, distorted vowels, vocal fry, } \\
\text { voicing breaks; spastic-predominant mixed dysarthria }\end{array}$} \\
\hline $\mathrm{A} 2, \mathrm{M}, 46$ & $\begin{array}{l}\text { Ms post-onset } \\
\text { ALSFRS-R } \\
\text { Intelligibility } \\
\text { Speech Characteristics }\end{array}$ & $\begin{array}{l}15 \\
41 \\
92.7 \\
\text { Dysarthri } \\
\text { phrases, i } \\
\text { breathy, }\end{array}$ & $\begin{array}{l}17 \\
40 \\
91.8 \\
\text { y perceivec } \\
\text { se articulat } \\
\text { listortions; }\end{array}$ & $\begin{array}{l}20 \\
28 \\
60.9 \\
\text { ssions 1-3) } \\
\text { (labial and } \\
\text { id-predom }\end{array}$ & $\begin{array}{l}\text { rate, short } \\
\text { nixed dysarthria }\end{array}$ \\
\hline A3, M, 49 & $\begin{array}{l}\text { Ms post-onset } \\
\text { ALSFRS-R } \\
\text { Intelligibility } \\
\text { Speech characteristics }\end{array}$ & \multicolumn{3}{|c|}{$\begin{array}{l}\text { Asymptomatic (Sessions 1-2), progressed to imprecise } \\
\text { articulation, hypernasality, vowel distortions, breathy, vocal fry } \\
\text { (Sessions 3-4); mixed spastic-flaccid dysarthria }\end{array}$} & $\begin{array}{l}31 \\
24 \\
38.2 \\
\text { ecise } \\
\text { athy, vocal fry }\end{array}$ \\
\hline
\end{tabular}


cake, and wait) containing high and low vowels were analyzed. These words were used in order to elicit a wide range of lip and jaw movement magnitudes.

\subsection{Recording procedures}

Speakers were positioned in a comfortable chair with head support. A 3D motion capture system (Motion Analysis Corporation-Eagle Cameras) was used to track movements of 15 small (approximately 3$4 \mathrm{~mm}$ ) sphere-shaped reflective markers attached to each speaker's head and face at specific anatomical landmarks (see top panel of Figure 1). Movements of these markers were recorded with eight infrared video cameras at 120 samples per second. Only markers attached to the vermilion borders of the upper and lower lips at midline (UL and LL) and the right side of the chin (thereafter JR), located approximately $2 \mathrm{~cm}$ off the chin midline were selected for this study. These markers are identified on the top portion of Figure 1.

During post-processing, movements of the facial markers were checked for tracking errors, low-pass filtered at $10 \mathrm{~Hz}$ using a zero-phase shift forward and reverse digital filter (8-pole Butterworth). Subsequently, the movements were head-corrected based on the subtraction of both the translational and rotational components of head movements. Positions of the markers were then re-expressed relative to the head-based coordinate system with the origin at the center of the mouth on the intersection of the planes passing through the corners of the mouth and the UL and center of the chin markers. The jaw movements were not subtracted from the lower lip movements because of concerns regarding the propagation of errors related to tracking the flesh of the chin (Green, Wilson, Wang, \& Moore, 2007). Thus all lip signals included those of the jaw (LL $+J)$.

The lip aperture history was used to identify the onset and offset of each measured interval. A lip aperture history calculated as a 3D Euclidean distance between the UL and LL markers recorded during the sentence Fast foxes have food feasts by subject A6 is shown in the bottom portion of Figure 1. The measurement boundaries are shown on the plot as black vertical lines; they are placed at the time when the lips are
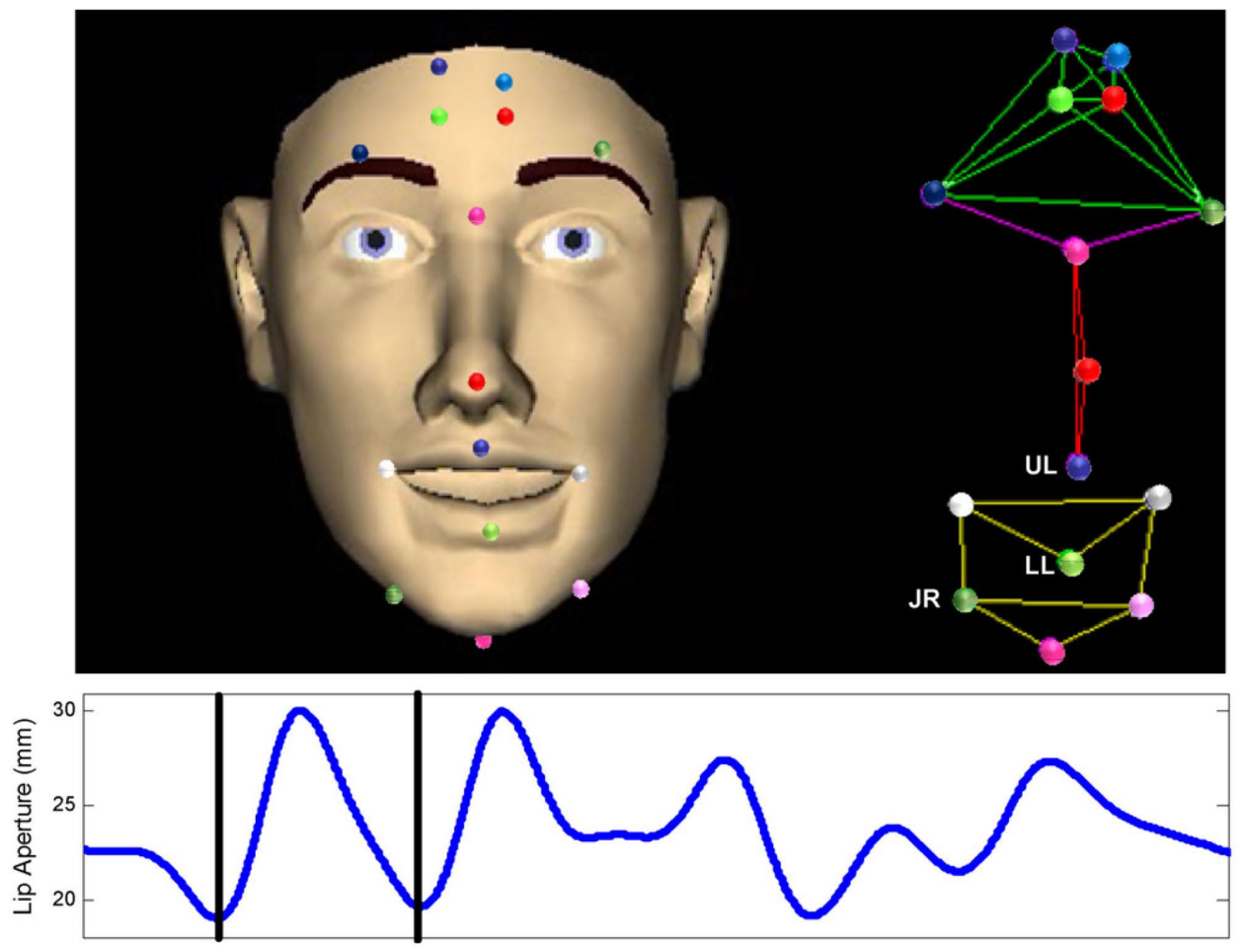

Figure 1. Top panel: The 15 marker model used for collecting facial kinematics. Only makers identified as UL (upper lip), LL (lower lip), and JR (jaw right) were used for this study. Bottom panel: Lip aperture trace in the sentence Fast foxes have food feasts produced by A6 during session 1. Vertical lines mark the boundaries of measured intervals. 
at the point of minimal opening during the initial and final consonants in fast. Measurements of UL, LL and JR movements during this interval were obtained (see below).

Acoustic signals were recorded simultaneously with kinematic signals directly onto a hard drive of a computer at the sampling rate of $32 \mathrm{KHz}$ and 16 bit resolution. A high quality lapel microphone (AudioTechnica AT831R) was mounted on a motion marker array, which was positioned on the forehead approximately $15 \mathrm{~cm}$ from the mouth during the recordings.

\subsection{Measurements}

Speech intelligibility and speaking rate were obtained for each subject and session using the Sentence Intelligibility Test (SIT; Beukelman, Yorkston, Hakel, \& Dorsey, 2007). Speaking rate was calculated as the number of words produced per minute. Intelligibility was expressed as the percent of total words transcribed correctly by a single judge, an unfamiliar listener. The listener was a healthy 35-year-old female with negative history of speech, language or hearing disorders. She was employed as a research assistant at the laboratory and had no formal training in motor speech disorders or experience with dysarthria.

Kinematic measures were selected based on prior studies of dysarthria demonstrating reduced size, slower speed, and longer durations of speech movements (Hirose et al., 1982; Yunusova et al., 2008). The following four kinematic measures were derived from the jaw (JR), upper lip (UL), and lower lip + jaw $(\mathrm{LL}+\mathrm{J})$ movements that were associated with the five target words.

(1) Path distance traveled by each marker during each word, a measure of movement size, was calculated as a sum of the 3D Euclidian distances between each time sample of the movement trajectory.

(2) Range of the first, second and third principal components of motion (P1, P2 and P3) of each movement trajectory was used to quantify changes in the overall geometry of speech movements. This measure was anticipated to be sensitive to deviations from the typical, vertically oriented trajectories of the jaw movements observed during speech (Tasko \& McClean, 2004). The P1 and P2 measures are shown in Figure

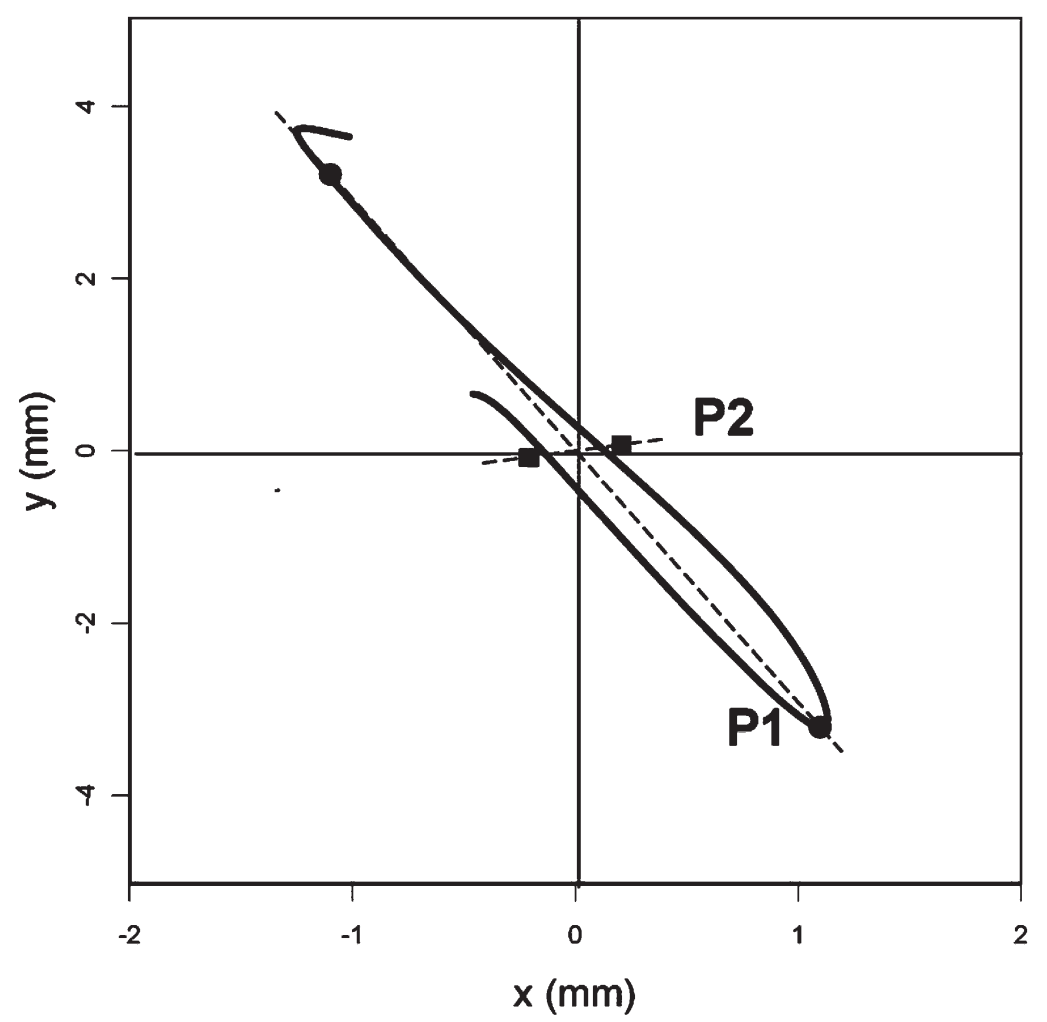

Figure 2. A jaw movement trajectory recorded during word fast produced by A6. P1 and P2 are the principal component axes for the first and second principal components, respectively. They are shown as dashed lines. 
2, where a representative jaw movement trajectory is plotted in the two-dimensional space during the word fast produced by A6. The principal component axes (P1 and P2) are shown as dashed lines. The ranges of P1 and P2 plotted as filled circles and squares, respectively, were calculated using the prcomp function in S-Plus 6.2 (Insightful Co, 2004).

(3) Average speed was calculated as the mean value of the first derivative of the $3 \mathrm{D}$ Euclidean distance movement history.

(4) Duration of the lip opening-closing gesture was calculated for the interval between movement onset and movement offset for each word (see bottom panel of Figure 1).

\subsection{Statistical analysis}

Change over time for the 5 words was assessed for each of the responses, markers, and subjects using a linear mixed effects (LME) model where words were considered replicates and the intercept term was allowed to vary by word. Time (month) was included as a categorical predictor so that no specific model was imposed on the pattern of the responses over time. The $p$-value for the initial analyses was adjusted for the number of responses and the number of subjects using a Bonferroni approach. For the LME analyses, paired comparisons were done by fitting additional LME models excluding all but the pair of months of interest. This approach is analogous to the protected $t$-tests method of paired comparison used in ANOVA.

\section{Results}

\subsection{Intelligibility and speaking rate}

Figure 3 shows speech intelligibility and speaking rate scores for each participant across recording sessions. Decline in both measures over time can be noted for all speakers. During the first three recording sessions (first two for A2), decline in intelligibility was relatively small, $5 \%$ on average for each of these speakers. The period of relatively stable intelligibility was followed by a precipitous drop in intelligibility between the last two recording sessions by 31,34 and $59 \%$ for speakers A1, A2, and A6, respectively.

Speaking rate scores showed a gradual decline across all sessions for speaker A1 $(19 \% \rightarrow 9 \% \rightarrow 21 \%)$. For speakers A2 and A6, a sizable decline in speaking rate was observed prior to the last recording session (57 and $46 \%$, respectively) followed by a plateau (5 and $9 \%$ change, respectively).

\subsection{Movement measures: jaw}

The movements of the upper lip were very small across contexts and did not show significant changes over the course of disease progression in any of the participants and, therefore, were not included in the summaries below. The P2 and P3 measures were also not included in the results because they either did not change over time (as in LL + J) or paralleled the results for the P1 measure (as in J). Table 2 summarizes results of analyses for $\mathrm{J}$.

\subsubsection{Path distance}

Statistically significant change in path distance were observed for speakers A1 $(F(3,12)=13.47, p<0.0068)$ and A2 $(F(2,8)=37.79, p<0.0015)$. For speaker A1, statistically significant changes occurred between months 1 and $6(t(4)=-6.07, p<0.0037), 1$ and $9(t(4)=-5.22, p<0.0064)$, and 9 and $12(t(4)=10.14$, $p<0.0005)$. For speaker A2, month 5 was significantly different from the preceding $(t(4)=9.92, p<0.0006)$ and the following months $(t(4)=7.67, p<0.0016)$.

\subsubsection{P1}

The size of the movement along the first principal component changed over time for all three speakers $(\mathrm{A} 1: F(3,12)=14.15, p<0.006$; A2: $F(2,8)=34.03, p<0.002 ; \mathrm{A} 6: F(3,12)=19.14, p<0.001)$. For A1, significant contrasts were obtained between months 1 and 6, 1 and 9, 6 and 12, and 9 and 12. For A2, results for the middle session differed from results for the preceding and following sessions. For A6, results for months 1, 3 and 9 differed from month 6 (see statistical summary in Table 3). 

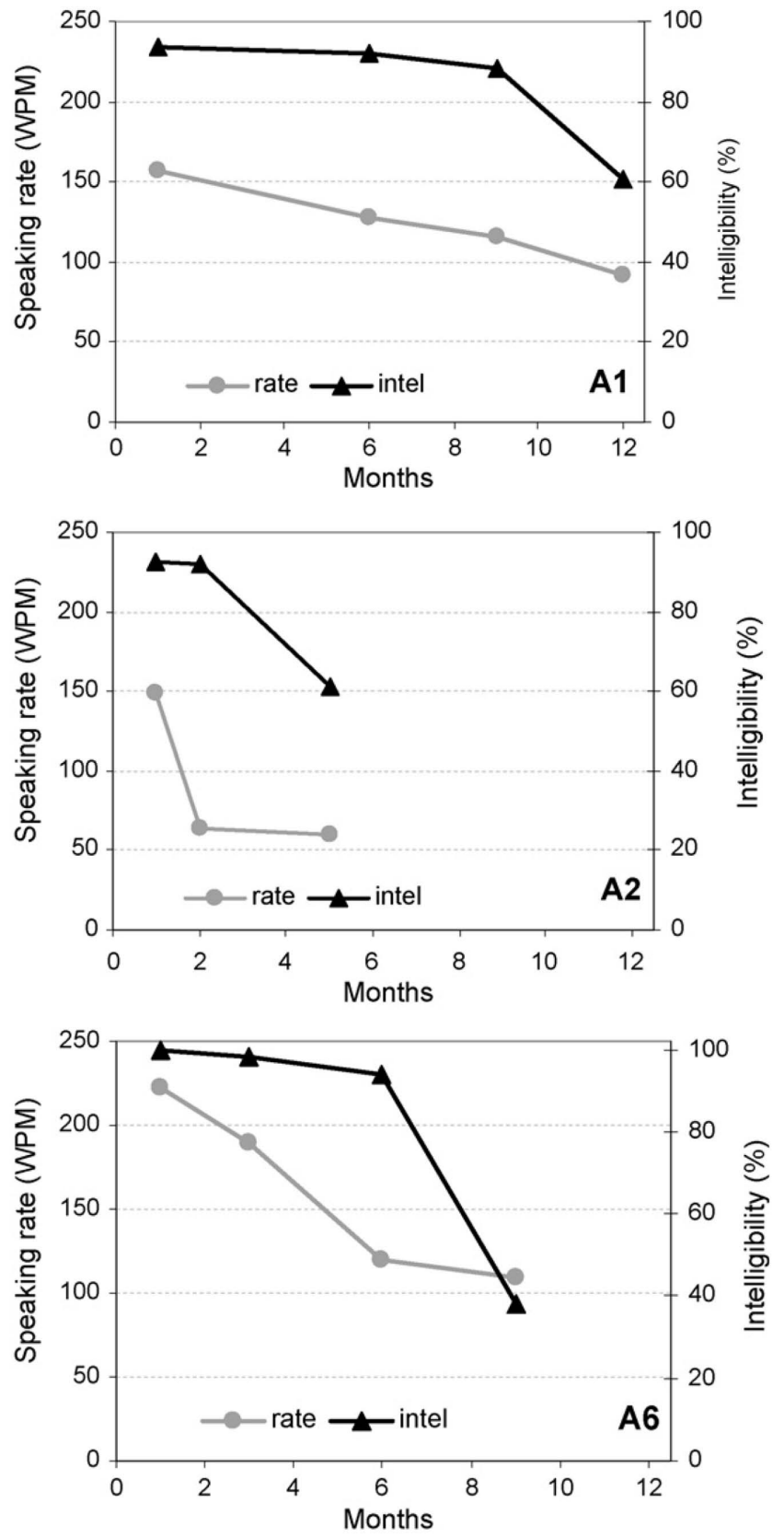

Figure 3. Speech intelligibility and speaking rate obtained for each speaker using the sentence intelligibility test. Months since the beginning of the study are indicated on the $X$-axes.

\subsubsection{Average speed}

Significant changes in average speed over time were observed for speakers A1 $(F(3,12)=35.98, p<0.001)$ and A2 $(F(2,8)=56.81, p<0.0003)$. For A1, month 1 differed from month $6(t(4)=-12.66, p<0.0002), 9$ $(t(4)=-9.87, p<0.0006)$ and $12(t(4)=-3.43, p<0.026)$. A statistical difference was also observed between months 9 and $12(t(4)=5.78, p<0.0045)$ for A1. For A2, statistically significant contrasts were between month 2 and months 1 and 5, respectively $(t(4)=11.91, p<0.0003$ and $t(4)=-10.2, p<0.0005)$.

\subsection{Movement measures: lower lip + jaw}

Table 4 shows summaries of kinematic measures obtained for LL $+\mathrm{J}$. 
Table 2. Means and standard deviations of selected kinematic measures for J. The measures that changed significantly over time are marked by an asterisk; a subscript indicates the months at which there were statistically different contrasts.

\begin{tabular}{lllll}
\hline A1 & $\mathbf{1}$ month & $\mathbf{6}$ months & $\mathbf{9}$ months & $\mathbf{1 2}$ months \\
\hline Path distance* $^{*}$ P $^{*}$ & $10.16(4.1)_{6,9}$ & $6.4(2.9)_{1}$ & $5.8(2.6)_{1,12}$ & $8.9(4.0)_{9}$ \\
Speed $^{*}$ & $4.6(1.7)_{6,9}$ & $3.2(1.4)_{1,12}$ & $3.4(1.7)_{1,12}$ & $5.1(2.4)_{6,9}$ \\
A2 & $20.4(4.9)_{6,9,12}$ & $12.2(3.5)_{1}$ & $11.7(4.4)_{1,12}$ & $15.6(6.4)_{1,9}$ \\
Path distance* & $\mathbf{1}$ month & $\mathbf{2}$ months & $\mathbf{5}$ months & \\
P1 $^{*}$ & $18.6(9.5)_{2}$ & $28.3(8.3)_{1,5}$ & $20.0(5.6)_{2}$ & \\
Speed* & $9.5(2.9)_{2}$ & $14.6(3.8)_{1,5}$ & $10.7(2.9)_{2}$ & \\
A6 & $42.9(15.0)_{2}$ & $64.8(20.0)_{1}$ & $35.6(9.2)_{2}$ & \\
Path distance & $\mathbf{1}$ month & $\mathbf{3}$ months & $\mathbf{6}$ months & $\mathbf{9}$ months \\
P1 & $13.3(6.2)$ & $13.4(5.8)$ & $16.4(6.2)$ & $14.3(5.7)$ \\
Speed & $7.2(3.1)_{6}$ & $7.0(3.0)_{6}$ & $3.3(1.7)_{1,3,9}$ & $7.5(3.1)_{6}$ \\
\hline
\end{tabular}

\subsubsection{Path distance}

The LL + J path distance measure did not change significantly between sessions for any of the speakers.

\subsubsection{P1}

Changes over time in P1 were statistically significant for A1 $(F(3,12)=12.2, p<0.01)$ and A6 $(F(3,12)=24.6$, $p<0.0004)$, resembling results for the J measure. For A1, session 1 was statistically different from each subsequent session ( 1 and $6: t(4)=-4.1, p<0.01 ; 1$ and $9: t(4)=-13.9, p<0.0002$; and 1 and $12: t(4)=-2.85$, $p<0.046$ ). For A6, session 3 (month 6) was statistically different from three other sessions (6 and 1: $t(4)=-4.76, p<0.009 ; 6$ and $3: t(4)=-4.98, p<0.008$; and 6 and $9: t(4)=8.64, p<0.0001)$.

\subsubsection{Average speed}

The speed of LL + J movements did not change significantly across sessions for A1. For A6, the measure changed significantly across recording sessions $(F(2,8)=9.21, p<0.035)$, with movement in month 9 (session 3) being significantly faster than all other months (1 and 9: $t(4)=4.22, p<0.013 ; 6$ and 9: $t(4)=3.6$, $p<0.02$; and 12 and 9: $t(4)=-4.94, p<0.008)$. For A2, the average speed of LL + J differed significantly between sessions $(F(2,8)=19.0, p<0.02)$. LL $+\mathrm{J}$ movements recorded during session 2 were faster than those during sessions $1(t(4)=4.37, p<0.01)$ and $3(t(4)=-6.51, p<0.003)$.

Independent contributions of the LL to the LL + jaw movement signals were examined through graphical analyses. Figure 4 shows an example of such an analysis for average speed. The change over time in the articulatory speed of the jaw for each speaker is shown with connected lines; the change in the LL + J movement speed is represented by dashed lines. Note, that for A1 and A6, contribution of the LL to the oral closure remained relatively similar across sessions. For A2, the jaw contribution to the LL appeared to increase for the middle session as compared to the initial and final sections (Figure 4).

Table 3. Summary of the LME results for the P1 measures for J. Only statistically significant contrasts are shown.

\begin{tabular}{lllcc}
\hline Subject & Months & DF & $t$-Value & $p$-Value \\
\hline A1 & $1-6$ & 1,4 & -5.44 & 0.006 \\
& $1-9$ & 1,4 & -7.35 & 0.002 \\
& $6-12$ & 1,4 & 3.34 & 0.029 \\
& $9-12$ & 1,4 & 4.55 & 0.011 \\
A2 & & & & \\
& $1-2$ & 1,4 & 8.28 & 0.001 \\
A6 & $2-5$ & 1,4 & -5.07 & 0.007 \\
& $1-6$ & 1,4 & -4.87 & 0.008 \\
& $3-6$ & 1,4 & -4.6 & 0.010 \\
\hline
\end{tabular}


Table 4. Means and standard deviations of kinematic measures for LL + J and duration measures. The measures that changed significantly over time are marked by an asterisk; a subscript indicates the months at which statistically different contrasts occurred.

\begin{tabular}{|c|c|c|c|c|}
\hline A1 & 1 month & 6 months & 9 months & 12 months \\
\hline Path distance & $14.9(4.4)$ & $11.3(4.1)$ & $9.5(3.9)$ & $11.1(4.2)$ \\
\hline $\mathrm{P} 1^{*}$ & $7.6(1.6)_{6,9,12}$ & $6.0(1.7)_{1}$ & $5.4(1.8)_{1}$ & $6.3(2.3)_{1}$ \\
\hline Speed & $30.5(6.9)^{-12}$ & $22.8(6.4)$ & $19.9(7.8)$ & $19.6(6.7)$ \\
\hline Duration & $520.8(125.4)$ & $526.0(180.4)$ & 514.0 (98.7) & $597.5(126.0)$ \\
\hline A2 & 1 month & 2 months & 5 months & \\
\hline Path distance & $27.2(7.8)$ & $32.2(9.6)$ & $29.1(7.9)$ & \\
\hline P1 & $15.0(3.9)$ & $17.1(4.6)$ & $15.5(3.7)$ & \\
\hline Speed* & $63.0(21.4)_{2}$ & $74.1(24.0)_{1,5}$ & $52.6(15.5)_{2}$ & \\
\hline Duration* & $467.5(87.7)$ & $454.4(70.0)_{1,5}$ & $578.0(92.3)_{2}$ & \\
\hline A6 & 1 month & 3 months & 6 months & 9 months \\
\hline Path distance & $20.9(7.6)$ & $20.8(7.5)$ & $25.5(6.9)$ & $23.9(7.2)$ \\
\hline $\mathrm{P} 1^{*}$ & $11.4(4.1)_{6}$ & $10.9(3.7)_{6}$ & $5.4(1.8)_{9}$ & $13.0(3.5)_{6}$ \\
\hline Speed* & $46.0(14.6)_{6}$ & $45.7(14.4)_{6}$ & $63.5(19.7)_{9}$ & $49.5(18.6)_{6}$ \\
\hline Duration & $476.0(99.9)$ & $450.8(80.5)$ & $420.8(66.8)$ & $515.2(95.6)$ \\
\hline
\end{tabular}

\subsection{Movement duration}

Summary of durational measures are presented in Table 4. Statistically significant change in the duration of an opening-closing cycle was observed for A2 only $(F(2,8)=17.37, p<0.02)$. For this participant, movement duration was longer for the last session at 5 months as compared to the first two recording sessions at 1 and 2 months $(t(4)=4.11, p<0.015$ and $t(4)=15.24, p<0.0001)$.

\subsection{Word effect}

Figure 5 shows an example of the changes over time in the measure of average jaw speed across different words. Although these data show that changes over time are similar across words, the observed effect appears to be greater for words with faster movements. A similar pattern of across word results were observed for other movement measures.

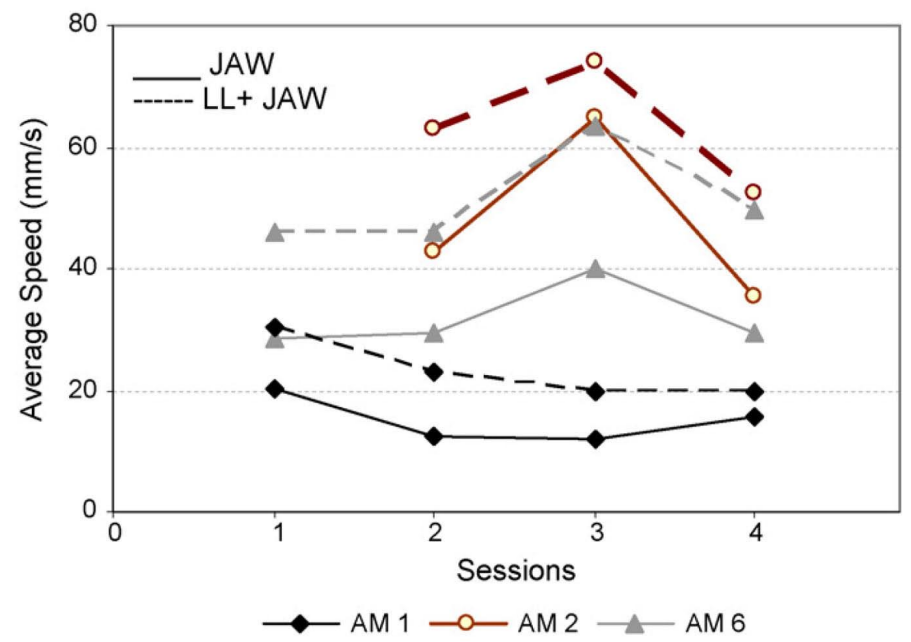

Figure 4. Average speed measures of the lower lip are represented by the dashed lines. The same measures obtained from the jaw are represented by the solid lines. 

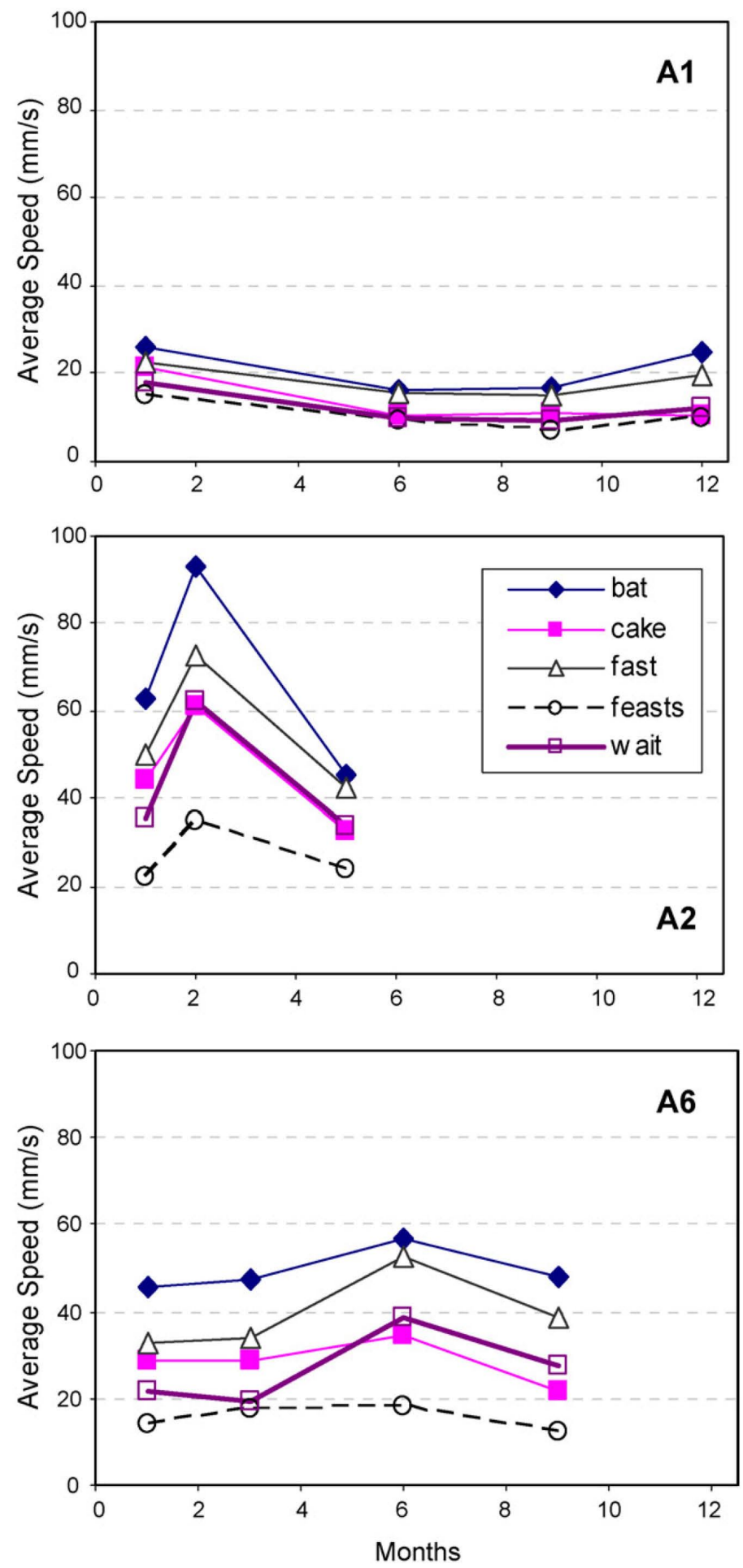

Figure 5. Word effect for J average speed over time for each speaker.

\subsection{Intelligibility $\sim$ speaking rate $\sim$ kinematic measures}

The relations between kinematic and perceptual measures were explored graphically. Figure 6 shows changes in intelligibility, speaking rate, and kinematic measures of the jaw over the course of the study for the three participants. Each variable is expressed as a percent relative to its value obtained on the first 

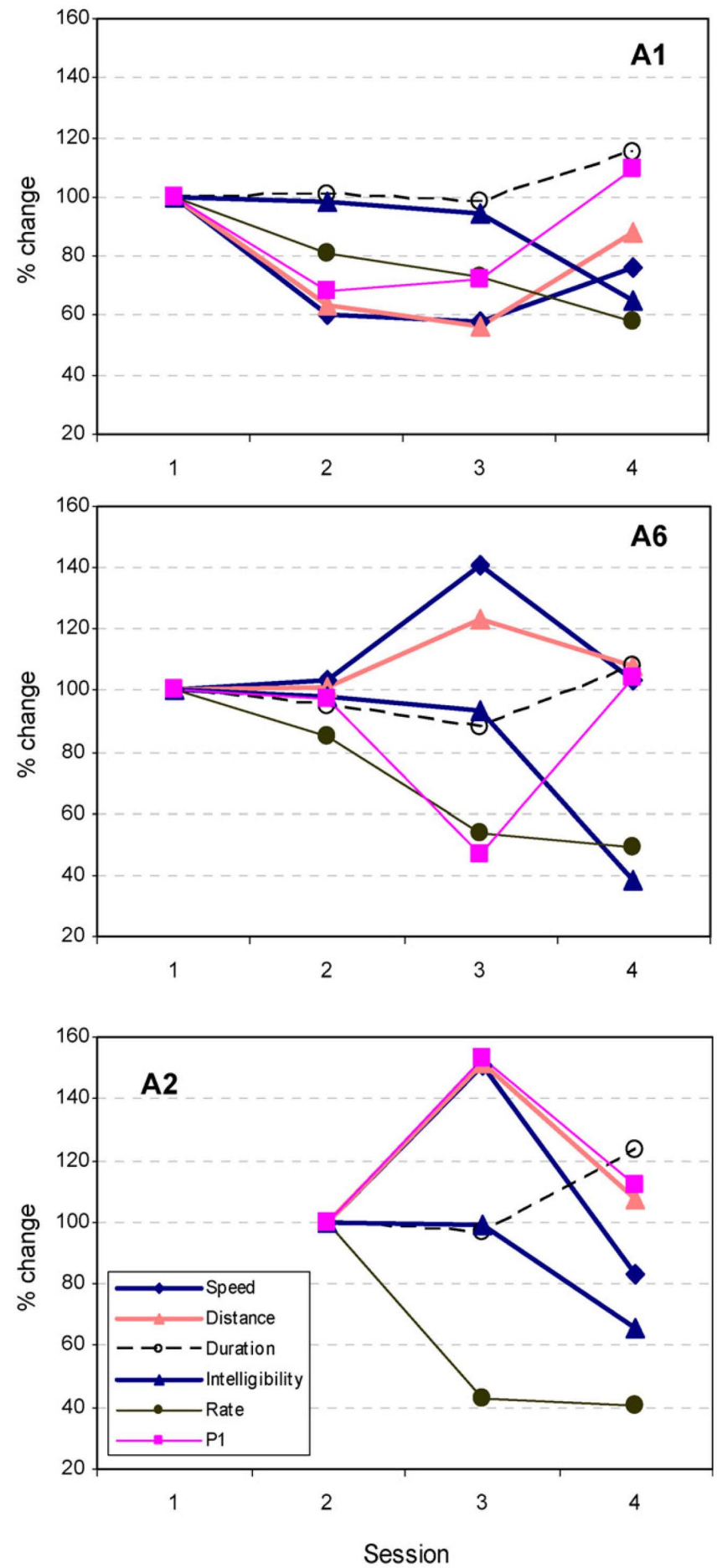

Figure 6. ALS progression as captured by jaw movement measures, speech intelligibility and speaking rate. The measures are expressed in percent change as calculated relative to the value obtained during the first recording session. $Y$ axis is in percent.

session. In all three cases, intelligibility dropped notably between the last two sessions. Speaking rate appeared to decline more gradually than did speech intelligibility, at least for speakers A1 and A6. The duration of the jaw opening and closing gesture remained relatively unchanged until the last session for each participant. Between the last two sessions, movement duration increased up to approximately $20 \%$. 
Kinematic measures (path distance, speed, and P1) showed patterns of change similar to each other, with the exception of P1 for A6. This measure decreased significantly in the third session, when the measures of path distance and speed increased during the same time interval. The significant increase (20-50\%) in the path distance and speed measures observed during the second to last session for speakers A2 and A6 corresponded to the largest decrease in speaking rate for these two speakers, while speech intelligibility remained stable. In speaker A1, the measures of path distance and speed declined up to $40 \%$ between sessions 1 and 2. These measures returned to near baseline between sessions 3 and 4 .

\section{Discussion}

Movements of the jaw and lower lip + jaw, speech intelligibility, and speaking rate were studied longitudinally in three individuals diagnosed with bulbar ALS. The primary objective of this study was to explore the relations among these variables with disease progression.

\subsection{Speech intelligibility, speaking rate and disease progression}

Speakers A1 and A6 entered the study when the effects of the disease on their speech was almost imperceptible. Speaker A2 entered the study when his speech was fully intelligible but already mild-to-moderately dysarthric (his ALS progressed very rapidly, resulting in a tracheotomy placement soon after the last recording session.) Initial high speech intelligibility was followed by a precipitous drop in intelligibility scores between the last two recording sessions for all speakers. Speaking rate at the beginning of the study was 250 WPM for A6 and slower than that, about 150 WPM, for A1 and A2. Speaking rate declined relatively gradually throughout the study for A1 and A6. For A2, who was in a relatively late stage of the disease, speaking rate dropped sharply within a short time interval between sessions 1 and 2 . As predicted by Yorkston et al. (1993) and Ball et al. (2002), speaking rate began to decline earlier than speech intelligibility for all three speakers. For A1 and A6, speech intelligibility declined significantly and precipitously within 3 months after the speaking rate reached 100-120 WPM. It dropped to 64 WPM before intelligibility declined for A2. Overall the data obtained from the participants in this study confirmed that speaking rate was more sensitive to bulbar disease progression than was speech intelligibility. Currently it is not known if these changes in speaking rate are primarily due to physiologic changes in the speech musculature (e.g., muscle weakness, fatigue) or whether they are compensatory in nature.

\subsection{Movement measures and disease progression}

Our findings were consistent with the results of previous cross-sectional research showing abnormal jaw and lower lip function in persons with ALS (Hirose et al., 1982; Yunusova et al., 2008). Specifically, movements became longer in duration. Jaw movements increased in size and speed at some point in time for all speakers. In A2 and A6, these increases occurred during the sessions that immediately preceded the session when speech intelligibility declined. Speaker A1's pattern of change differed from that of the other speakers. Specifically, his articulatory speed and path distance declined steadily over the 9 months of the study, which was followed by a significant increase in movement size and speed between the last two sessions. The current findings underscore the need to track bulbar decline for a longer interval than a year. Follow up studies are now being conducted that investigate patients for up to 2 years to ensure that none of the salient landmarks are missed.

The change in P1 was in an opposite direction from the trend observed for the speed and path distance measures for A6, suggesting a change in the shape of the movement trajectories for this speaker. This finding was confirmed on observations of individual jaw trajectories obtained during session 3 for A6. However, the same was not observed for any other speakers. Weismer et al. (1992) reported qualitatively different F1 and F2 trajectories in vowels produced by severely impaired speakers with ALS, suggesting difficulties in transitioning between segments (i.e., segmentalization or movement decomposition) in these speakers. Studies of spastic limb movements have also shown a pattern of decomposition among movement elements (Steenbergen and Meulenbroek, 2003). Movement decomposition might alter the shape of movement trajectories beyond changes in their size. These findings suggest the need for more comprehensive investigations of topologic changes in articulatory movements in ALS. 


\subsection{Movement measures, speech intelligibility, and speaking rate}

The increase in movement duration between the last two sessions demonstrated by each speaker coincided with a precipitous drop in their speech intelligibility during the same time interval. Only for A1 was a notable change in speaking rate $(21 \%)$ observed during the same time interval. This finding raises the possibility that, early in the disease, the slowing of speaking rate is due to an increase in the frequency and the duration of pauses (Green, Ball, \& Beukelman, 2004). Interestingly, the speaking rate reduction just prior to the last session was associated with higher speed and size of jaw and LL + J movements. One possible explanation for these contradictory results is that the slowing of speaking rate beyond 100-120 WPM is associated with the slowing of tongue movements, and that speaking rate is still decreasing because of increases in pause duration and frequency (see Weismer et al., 2001 for a similar discussion).

For speakers A2 and A6, a rapid increase in jaw speed and path distance during the second to last session preceded the precipitous drop of speech intelligibility in the last session. This dramatic increase in jaw movement might be a response to a reduction in tongue movement. This observation is consistent with interpretations made by several other investigators (Hirose et al., 1982; DePaul and Abbs, 1987). However, strong empirical evidence of compensatory articulation based on simultaneous recordings of the jaw and tongue is still lacking.

For two out of three speakers, jaw kinematics anticipated the change in speech intelligibility by 3 months, when speaking rate decline was more gradual. This might suggest that kinematic variables may be better predictors of intelligibility decline than speaking rate and should be monitored during the course of the disease.

\subsection{Differential impairment: jaw and lower lip}

The differential degree of impairment among the different components of the speech mechanism makes ALS an interesting condition to study for understanding the physiologic basis of speech impairment. Our data, however, showed minimal evidence of disassociation between the effect of ALS on jaw and lower lip performance. The graphical analysis showed a slight increase in the contribution of the jaw to LL $+\mathrm{J}$ movements in $\mathrm{A} 2$ at a middle session. Otherwise, the disease-related changes in the lower lip were nearly identical to those observed in the jaw. This finding might be explained, in part, by the characteristics of the stimuli used in this study of which only one word (wait) was a bilabial glide requiring specific labial adjustments to produce its acoustic target. Selecting words that stress the labial mechanism should be considered in the future to elicit unique lip behaviors. However, the minimal changes in the LL is also consistent with the observation that the jaw and lip musculature are less affected by ALS than the tongue musculature, with neuroanatomical data confirming this difference at the level of hypoglossal, facial, and trigeminal nuclei (DePaul and Brooks, 1993; DePaul and Abbs, 1987; Lawyer and Netsky, 1953). Yunusova et al. (2008) reported no changes in the size of lip movements recorded during vowels in a group of speakers with ALS. Slower than normal movements of the lower lip in these patients were attributed to a compensatory response in the lips, working to preserve timing relationships between the articulators involved in vowel production. The findings of this study support these observations.

\subsection{Individual differences}

Individual differences in the pattern of disease progression are not well understood (see Yorkston et al., 1998). In the current investigation, a precipitous pattern of decline in intelligibility was similar across speakers, whereas the pattern of speaking rate and articulatory performance decline varied notably. For example, speaking rate declined steadily for A1 during the 12 months of the study. In contrast, speaking rate dropped significantly within the first 2 months in the study for A2 and dropped more gradually within the first 6 months for A6. These two participants also showed a pattern of jaw and lower lip movement change that was different from A1. It is notable that A2 and A6 were judged perceptually as having a more predominant flaccid component in their dysarthria than A1 whose speech had predominantly spastic features. Additionally, Yorkston, Strand, and Hume (1989) suggested that speech intelligibility may vary across individuals with dysarthria depending on the subsystems that are preserved (e.g., relatively preserved respiratory-phonatory subsystem and the jaw might be associated with better speech intelligibility), the rate of disease progression, and the cognitive status of an individual. Additional studies are needed to elucidate the relations between functional outcomes of ALS and the variety of physiologic factors that might play a role in affecting the speech function. 


\subsection{Conclusions}

This small $\mathrm{N}$ longitudinal study was designed to explore the effects of ALS on kinematic measures of speech and their relationship to measures of speech intelligibility and rate. The results showed that jaw and lip movement measures changed over time, with movement duration closely mimicking the pattern of intelligibility decline. Because movement measures tended to change from baseline prior to the drop in speech intelligibility, these measures may prove to be predictive of intelligibility declines and may therefore be useful clinical markers for initiation of compensatory interventions such as AAC. However, speaking rate, which tended to decline earlier than the movement measures in all three speakers, might be a good marker for detecting the onset of bulbar symptoms. The current findings support prior suggestions that speaking rate is a good clinical indicator of overall bulbar deterioration, particularly considering that it is also so easy to obtain. The preliminary findings of this study need to be expanded in the future to the investigation of multiple speech subsystems in a larger number of participants.

\section{Acknowledgments}

This research was supported by NIH-NIDCD Grant \#1R01DC009890-01A1 and Barkley Trust. We would like to thank Cynthia Didion and Cara Ullman for assistance in data collection and management.

\section{References}

Ball et al., $2002-$ L. J. Ball, D. R. Beukelman, and G. L. Pattee, Timing of speech deterioration in people with amyotrophic lateral sclerosis, Journal of Medical Speech-Language Pathology 10 (2002), pp. 231-235.

Beukelman et al., 2007 D. Beukelman, K. Yorkston, M. Hakel, and M. Dorsey, Speech intelligibility test (Computer software), Madonna Rehabilitation Hospital, Lincoln, NE (2007).

Brooks et al., $2000 \rightarrow$ B. R. Brooks, R. G. Miller, M. Swash, and T. L. Munsat, El Escorial revisited: Revised criteria for the diagnosis of amyotrophic lateral sclerosis, Amyotrophic Lateral Sclerosis and Other Motor Neuron Disorders 1 (2000), pp. 293-299.

Brooks et al., 1991 B. R. Brooks, R. L. Sufit, R. DePaul, Y. D. Tan, M. Sanjak, and J. Robbins, Design of clinical therapeutic trials in amyotrophic lateral sclerosis, Advances in Neurology 56 (1991), pp. 521-546.

Caruso and Burton, 1987 A. Caruso and E. K. Burton, Temporal acoustic measures of dysarthria associated with amyotrophic lateral sclerosis, Journal of Speech and Hearing Research 30 (1987), pp. 80-87.

Cedarbaum et al., 1999 J. M. Cedarbaum, N. Stambler, E. Malta, C. Fuller, D. Hilt, B. Thurmond, and A. Nakanishi, The ALSFRS-R: A revised ALS functional rating scale that incorporates assessments of respiratory function, Journal of the Neurological Sciences 169 (1-2) (1999), pp. 13-21.

Czaplinski et al., 2006 A. Czaplinski, A. A. Yen, and S. H. Appel, Amyotrophic lateral sclerosis: Early predictors of prolonged survival, Journal of Neurology 253 (2006), pp. 1428-1436.

Darley et al., 1975 F. L. Darley, A. E. Aronson, and J. R. Brown, Motor speech disorders, WB Saunders, Philadelphia (1975).

DePaul and Abbs, $1987 \rightarrow$ R. DePaul and J. H. Abbs, Manifestations of ALS in the cranial motor nerves: Dynametric, neuropathologic, and speech motor data, Neurologic Clinics 5 (2) (1987), pp. 231-250.

DePaul et al., $1988 \longrightarrow$ R. DePaul, J. H. Abbs, M. P. Caligiuri, V. L. Gracco, and B. R. Brooks, Differential involvement of hypoglossal, trigeminal and facial motoneurons in ALS, Neurology 38 (1988), pp. 281-283.

DePaul and Brooks, $1993 \longrightarrow$ R. DePaul and B. R. Brooks, Multiple orofacial indices in amyotrophic lateral sclerosis, Journal of Speech and Hearing Research 36 (1993), pp. 1158-1167.

DePaul and Kent, $2000 \checkmark$ R. DePaul and R. D. Kent, A longitudinal case study of ALS: Effects of listener Familiarity and proficiency of intelligibility judgments, American Journal of Speech Language Pathology 9 (2000), pp. 230-240.

Dworkin, 1980 J. P. Dworkin, Tongue strength measurement in patients with amyotrophic lateral sclerosis: Qualitative vs. quantitative procedures, Archives of Physical Medicine and Rehabilitation 61 (1980), pp. 422-424.

Duffy, $2005 \longrightarrow$ J. R. Duffy, Motor speech disorders: Substrates, diagnosis, and management (2nd ed. ), Elsevier Mosby, St. Louis (2005).

Green et al., 2004 J. R. Green, D. R. Beukelman, and L. J. Ball, Algorithmic estimation of pauses in extended speech samples of dysarthric and typical speech, Journal of Medical-Speech Language Pathology 12 (4) (2004), pp. 149-154. 
Green et al., 2007 J. R. Green, E. M. Wilson, Y. Wang, and C. A. Moore, Estimating mandibular motion based on chin surface targets during speech, Journal of Speech, Language, and Hearing Research 50 (2007), pp. 928-939.

Hirose et al., 1982 H. Hirose, S. Kiritani, and M. Sawashima, Patterns of dysarthric movement in patients with amyotrophic lateral sclerosis and pseudobulbar palsy, Folia Phoniatrica et Logopaedica 34 (1982), pp. 106-112.

Kent et al., 1992 J. F. Kent, R. D. Kent, J. C. Rosenbek, G. Weismer, R. Martin, and R. Sufit, Quantitative description of the dysarthria in women with amyotrophic lateral sclerosis, Journal of Speech and Hearing Research 35 (4) (1992), pp. $723-733$.

Kent et al., 1989 R. D. Kent, J. F. Kent, G. Weismer, R. E. Martin, R. L. Sufit, and J. C. Rosenbek, Relationships between speech intelligibility and the slope of second-formant transitions in dysarthric subjects, Clinical Linguistics and Phonetics 3 (1989), pp. 347-358.

Kent et al., $1990>$ R. D. Kent, J. F. Kent, G. Weismer, R. L. Sufit, J. C. Rosenbek, and R. E. Martin, Impairment of speech intelligibility in men with amyotrophic lateral sclerosis, Journal of Speech and Hearing Disorders 55 (1990), pp. 721-728.

Kent et al., 1991 R. D. Kent, R. L. Sufit, J. C. Rosenbek, J. F. Kent, G. Weismer, and R. E. Martin, Speech deterioration in amyotrophic lateral sclerosis: A case study, Journal of Speech and Hearing Research 34 (6) (1991), pp. 1269-1275.

Langmore and Lehman, 1994 S. E. Langmore and M. E. Lehman, Physiologic deficits in the orofacial system underlying dysarthria in amyotrophic lateral sclerosis, Journal of Speech and Hearing Research 37 (1) (1994), pp. 28-37.

Lawyer and Netsky, $1953 \longrightarrow$ T. Lawyer and M. G. Netsky, Amyotrophic lateral sclerosis. A clinicoanatomic study of 53 cases, Archives of Neurology and Psychiatry 69 (1953), pp. 171-192.

Mulligan et al., 1994 M. Mulligan, J. Carpenter, J. Riddel, M. K. Delaney, G. Badger, and P. Krusinski, Intelligibility and the acoustic characteristics of speech in amyotrophic lateral sclerosis (ALS), Journal of Speech and Hearing Research Vol. 37 (1994), pp. 496-503.

Millul et al., 2005 A. Millul, E. Beghi, G. Logroscino, A. Micheli, E. Vitelli, and A. Zardi, Survival of patients with amyotrophic lateral sclerosis in a population-based registry, Neuroepidemiology 25 (2005), pp. 114-119.

Nishio and Niimi, $2000-$ M. Nishio and S. Niimi, Changes over time in dysarthric patients with amyotrophic lateral sclerosis (ALS): A study of changes in speaking rate and maximum repetition rate (MRR), Clinical Linguistics $\mathcal{E}$ Phonetics 14 (7) (2000), pp. 485-497.

Ramig et al., $1990 \rightarrow$ L. O. Ramig, R. C. Scherer, E. R. Klasner, I. R. Titze, and Y. Horii, Acoustic analysis of voice in amyotrophic lateral sclerosis: A longitudinal case study, Journal of Speech and Hearing Disorders 55 (1) (1990), pp. 2-14.

Steenbergen and Meulenbroek, $2003-$ B. Steenbergen and R. G. J. Meulenbroek, Signs of long-term adaptation to permanent brain damage as revealed by prehension studies of children with spastic hemiparesis. In: M. L. Latash and M. Levin, Editors, Progress in motor control III: Effects of age, disorder and rehabilitation, Human Kinetics, Champaign, IL (2003), pp. 207-234.

Tasko and McClean, 2004 S. M. Tasko and M. D. McClean, Variations in articulatory movement with changes in speech task, Journal of Speech, Language, and Hearing Research 47 (2004), pp. 85-100.

Tjaden and Turner, 2000 K. Tjaden and G. Turner, Segmental timing in amyotrophic lateral sclerosis, Journal of Speech, Language, and Hearing Research 43 (2000), pp. 683-696.

Tjaden and Turner, 1997 K. Tjaden and G. Turner, Spectral properties of fricative in amyotrophic lateral sclerosis, Journal of Speech, Language, and Hearing Research 40 (1997), pp. 1358-1372.

Turner et al., $1995 \rightarrow$ G. S. Turner, K. Tjaden, and G. Weismer, The influence of speaking rate on vowel space and speech intelligibility for individuals with ALS, Journal of Speech and Hearing Research 38 (1995), pp. 1001-1013.

Turner and Weismer, 1993 G. S. Turner and G. Weismer, Characteristics of speaking rate in the dysarthria associated with amyotrophic lateral sclerosis, Journal of Speech and Hearing Research 36 (6) (1993), pp. 1134-1144.

Weismer et al., 2001 G. Weismer, J. Y. Jeng, J. S. Laures, R. D. Kent, and J. F. Kent, Acoustic and intelligibility characteristics of sentence production in neurogenic speech disorders, Folia Phoniatrica et Logopaedica 53 (1) (2001), pp. 1-18.

Weismer et al., $1992>$ G. Weismer, R. Martin, J. F. Kent, and R. D. Kent, Formant trajectory characteristics of males with amyotrophic lateral sclerosis, Journal of the Acoustical Society of America 91 (1992), pp. 1085-1097.

Weismer et al., 1988 G. Weismer, R. D. Kent, M. Hodge, and R. Martin, The acoustic signatures for intelligibility test words, Journal of the Acoustical Society of America 84 (4) (1988), pp. 1281-1291.

Yorkston et al., $1993 \rightarrow$ K. M. Yorkston, E. Strand, R. Miller, A. Hillel, and K. Smith, Speech deterioration in amyotrophic lateral sclerosis: Implications for the timing of intervention, Journal of Medical Speech-Language Pathology 1 (1993), pp. 35-46.

Yorkston et al., $1998-$ K. M. Yorkston, E. A. Strand, and J. Hume, The relationship between motor function and speech function in amyotrophic lateral sclerosis. In: M. Cannito, K. M. Yorkston and D. R. Beukelman, Editors, Neuromotor speech disorders: Nature, assessment, and management, Paul H. Brookes, Baltimore (1998), pp. 85-98.

Yunusova et al., $2008 \longrightarrow$ Y. Yunusova, G. Weismer, J. R. Westbury, and M. Lindstrom, Articulatory movements during vowels in speakers with dysarthria and normal controls, Journal of Speech, Language, and Hearing Research 51 (3) (2008), pp. 596-611. 